\title{
Institutsvorstellung
}

\section{Die Neurowissenschaftliche Gesellschaft ist Gründungsmitglied des German Brain Council}

https://doi.org/10.1515/nf-2018-0015

Am 7. Februar 2018 trafen sich die Vertreter von 14 Fachgesellschaften der Neurowissenschaften aus Deutschland in Berlin, um das German Brain Council als eine nationale Unterorganisation des European Brain Councils (EBC, www.braincouncil.eu/) zu gründen. Das German Brain Council ist ein gemeinnütziger Verein, dessen Mitglieder wissenschaftliche Fachgesellschaften, Patientenorganisationen und Unternehmungen sein können, die laut Satzung ,auf dem Gebiet der Erforschung, Prävention, Diagnostik und Behandlung einschließlich Rehabilitation und Nachsorge von Erkrankungen des menschlichen Nervensystems tätig sind. Zweck des Vereins ist die Förderung von Wissenschaft und Forschung im Zusammenhang mit Erkrankungen des menschlichen Nervensystems. Der Verein hat die Aufgabe, Erkenntnisse, Entwicklungen und Aktivitäten auf diesem Gebiet zu bündeln und in gesellschaftliche, politische und Entscheidungsprozesse auf nationaler und europäischer Ebene einzubringen“. Das German Brain Council soll insbesondere die deutschen Interessen im European Brain Council vertreten und durch geeignete Öffentlichkeitsarbeit über Funktionen und Erkrankungen des menschlichen Nervensystems aufklären. Ein weiterer wichtiger Aspekt ist, bei politischen Verantwortungs- und Entscheidungsträgern für die Sache der Neurowissenschaften zu werben und sie von der Wichtigkeit der Forschung auf diesem Gebiet zu überzeugen. Innerhalb des German Brain Council wird die Neurowissenschaftliche Gesellschaft die „Stimme der Grundlagenforschung“ sein. Was es in der deutschen Neurowissenschaft zu erhalten gilt, ist ein enges Verhältnis der biologischen zu den medizinischen Wissenschaften und damit ein Verständnis für evolutive Zusammenhänge, die sich bis zur Erforschung grundlegender Mechanismen von Pathologien des menschlichen Gehirns in tierischen Nervensystemen erstrecken. Das German Brain Council versteht sich als ein übergeordnetes Bündnis der verschiedensten Mitgliedsgesellschaften, das gegenüber der Politik mit einer Stimme spricht und für die Erforschung der Erkrankungen des Nervensystems eintritt. In Zukunft sollen neben den 14 Gründungs-Fachgesellschaften noch weitere Mitglieder dazu kommen. Bis auf
Weiteres ist die Geschäftsstelle des German Brain Council bei der Deutschen Gesellschaft für Neurologie (DGN), Reinhardtstraße 27c, 10117 Berlin, untergekommen.

Hans-Joachim Pflüger, Berlin

Gründungsmitglieder des German Brain Council sind:

- Deutsche Gesellschaft für Neurologie (DGN)

- Deutsche Gesellschaft für Psychiatrie und Psychotherapie, Psychosomatik und Nervenheilkunde (DGPPN)

- Deutsche Gesellschaft für Neurochirurgie (DGCN)

- Deutsche Gesellschaft für klinische Neurophysiologie und funktionelle Bildgebung (DGKN)

- Deutsche Gesellschaft für Neuropathologie und Neuroanatomie (DGNN)

- Deutsche Gesellschaft für Neurorehabilitation (DGNR)

- Deutsche Gesellschaft für Kinder- und Jugendpsychiatrie (DGKJP)

- Neurowissenschaftliche Gesellschaft (NWG)

- Gesellschaft für Neuropädiatrie (GNP)

- Deutsche Gesellschaft für Neuroradiologie (DGNR)

- Deutsche Gesellschaft für Neuromodulation (DGNM)

- Deutsche Gesellschaft für Epileptologie (DGfE)

- Deutsche Gesellschaft für Parkinson- und Bewegungsstörungen (DPG)

- Deutsche Gesellschaft für Bipolare Störungen (DGBS)

Der Vorstand des German Brain Council besteht aus folgenden Personen:

- Präsident: Prof. Dr. Alfons Schnitzler, Düsseldorf

- Stellvertretender Präsident: Prof. Dr. Peter Falkai, München

- Schriftführer: Prof. Dr. Thomas Mokrusch, Lingen

- Schatzmeister: Priv.-Doz. Dr. Gerhard J. Jungehülsing, Berlin

- Beisitzer: Prof. Dr. Ulrike Schara, Essen

- Beisitzer: Prof. Dr. Dr. h. c. Wolfgang H. Oertel, Marburg

- Beisitzer: Prof. Dr. rer. nat. Hans-Joachim Pflüger, Berlin 


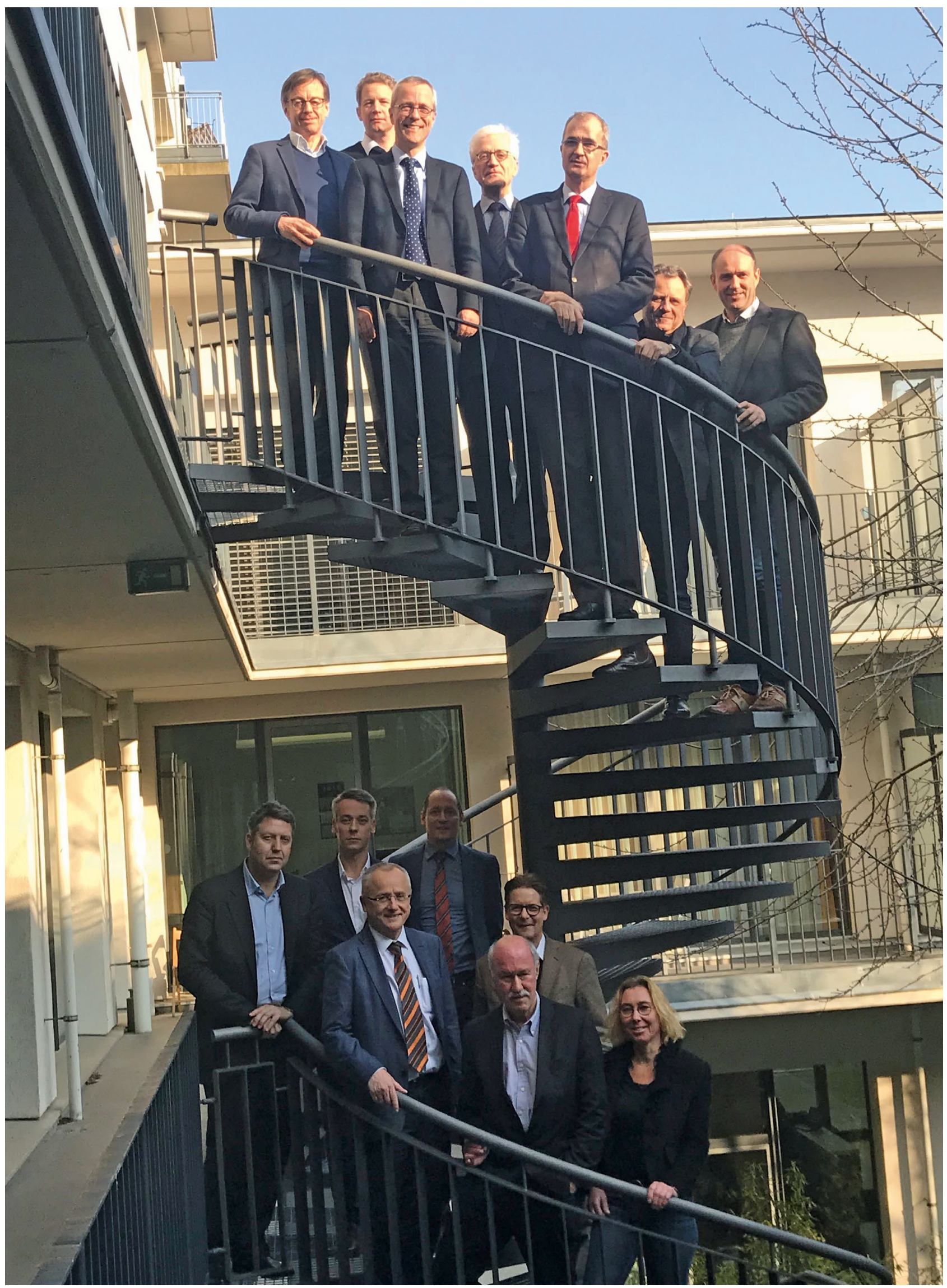

